\title{
True incidence of recurrent laryngeal nerve injury: time to audit!
}

Linked Comment: Jeannon. Int J Clin Pract 2010; 64: 523.

To the Editor:

Sir,

Dr Jeannon and associates (1) reported a comprehensive meta analysis on the postoperative diagnosis of recurrent laryngeal nerve (RLN) palsy after thyroid surgery. I have carefully read this excellent paper. Certainly, these colleagues have made several contributions to the literature, and their valuable contributions have advanced our understanding of the surgical technique in neck surgery. I would like to offer additional remarks.

- The authors demonstrated that there is a considerable variation in the reported frequency of RLN palsy rates after thyroid surgery because of the different methods of diagnosing RLN palsy (indirect laryngoscopy, fibre-optic nasolaryngoscopy and video-stroboscopy) each of which have significant different sensitivities and specificities (1). The rate of RLN paralysis is yet underestimated for other reasons: (i) no routine surgical Audit by Centers performing thyroid and parathyroid surgery, not all patients in fact undergo postoperative laryngeal examination (2); (ii) units with unfavourable data, are less likely to report their data $(3,4)$; (iii) lack of data in Literature on bilateral RLN paralysis incidence (3); (iv) lack of reliability of clinical symptoms in vocal cord paralysis (thus, the need of a routine postoperative laryngoscopy) $(3,4)$; (v) the incidence of RLN palsy may be influenced also by the 'timing' of a postoperative vocal cord check (2-4).
- The authors focused on the postoperative diagnosis of RLN palsy. At present, in the modern era of thyroid surgery, the diagnosis of RLN palsy might be also intra-operatively (and not postoperative) with the application of neuromonitoring (IONM) of the laryngeal nerves. The intra-operative assessment of RLN function with IONM during thyroid surgery is important for different reasons: intra-operative prediction of postoperative function (prognosis); prevention of bilateral RLN injury (stage thyroidectomy); identification of how the RLN was injured; early differentiation between RLN related and unrelated voice changes (5). I believe that IONM may clarify the true incidence of RLN injury. I do not mean to emphasise new technologies over sound common sense and I do recognise that at present, the standards for RLN management during thyroid surgery are yet extensive knowledge of RLN anatomy, routine visual identification of nerve, cervical exposure of RLN, experience, training and again pre- and postoperative laryngoscopy.

Thank you for the opportunity to write this letter.

G. Dionigi

Department of Surgical Sciences, Endocrine Surgery Research Center, University of Insubria, Varese, Italy Email: gianlorenzo.dionigi@uninsubria.it

\section{References}

1 Jeannon JP, Orabi AA, Bruch GA, Abdalsalam HA, Simo R. Diagnosis of recurrent laryngeal nerve palsy after thyroidectomy: a systematic review. Int J Clin Pract 2009; 63: 624-9.

2 Bergenfelz A, Jansson S, Kristoffersson A et al. Complications to thyroid surgery: results as reported in a database from a multicenter audit comprising 3,660 patients. Langenbecks Arch Surg 2008; 393: 66773.

3 Randolph GW. Surgical anatomy of the recurrent laryngeal nerve. In: Randolph GW, ed. Surgery of the Thyroid and Parathyroid Glands. Elsevier Science, Philadelphia, USA, 2003: 1123-67.

4 Steurer M, Passler C, Denk DM et al. Advantages of recurrent laryngeal nerve identification in thyroidectomy and parathyroidectomy and the importance of preoperative and postoperative laryngoscopic examination in more than 1000 nerves at risk. Laryngoscope 2002; 112: 124-33.

5 Dralle H, Sekulla C, Lorenz $\mathrm{K}$ et al. Intraoperative monitoring of the recurrent laryngeal nerve in thyroid surgery. World J Surg 2008; 32: 1358-66.

\section{Disclosures}

No competing interest.

${ }^{1}$ Commentary on the paper Diagnosis of recurrent laryngeal nerve palsy after thyroidectomy: a systematic review. By Jeannon JP, Orabi AA, Bruch GA, Abdalsalam HA, Simo R. Int J Clin Pract. 2009;63(4):624-9.

doi: $10.1111 / j .1742-1241.2009 .02193 . x$

\section{LETTER}

\section{Universal audit of thyroidectomy results is necessary}

\section{Linked Comment: Dionigi. Int J Clin Pract 2010; 64: 523.}

To the Editor:

Sir,

Thank you very much for the opportunity to respond to Dr Dionigi's commentary on our study. I am grateful to his kind comments, and I value the insightful and helpful observations made. I agree in his summary that so many other important factors not covered in our review play an important role in affecting rates of recurrent laryngeal nerve palsy following thyroidectomy.

Standardisation of procedure and reporting is necessary through universal audit of thyroidectomy results.
We did not investigate the impact of intraoperative nerve monitoring (IONM) during thyroidectomy in our study. We recognise that it is increasingly becoming an integral part of thyroid surgical practice in many centres. I have personal experience in using this technique for teaching purposes and for revision surgery. I am sure that IONM for thyroidectomy will become the standard of care in the future.

The importance of IONM in thyroidectomy could be demonstrated through a prospective randomised controlled trial or a systematic review.
J.-P. Jeannon

Ear Nose \& Throat Department, Guy's Hospital, London, UK Email: jpjeannon@doctors.org.uk 